\title{
Modeling Electromagnetic Interference Generated by a WLAN System Onboard Commercial Aircraft
}

\author{
Clifford De Raffaele ${ }^{1}$, Carl James Debono ${ }^{2}$ and Adrian Muscat ${ }^{3}$ \\ Department of Communications \& Computer Engineering \\ University of Malta, Msida, MSD2080, Malta \\ cderaffaele@ieee.org, ${ }^{2}$ c.debono@ieee.org, ${ }^{3}$ adrian.muscat@um.edu.mt
}

\begin{abstract}
The growing demand for the utilization of personal electronic communication devices onboard commercial aircraft necessitates the assurance of safety by airline operators and regulators. This implies that the potential risks posed by the deployment of wireless communication systems on critical aircraft equipment must be carefully assessed and countermeasures taken when required. In this paper, a model based on a ray-tracing algorithm is developed to calculate the electromagnetic interference incident on the fuselage structure of a commercial airline. The source of interference is a $2.4 \mathrm{GHz}$ data communications network. Two scenarios are considered; the first assumes a base station in the centre of the cabin while the second considers four base stations, transmitting at a lower power, distributed along the cabin. The model first determines the propagation map generated by the base stations. These results are used to establish the transmission power required by the personal mobile devices which is then employed to determine the propagation map of each device. The overall electromagnetic interference map incident on the fuselage resulting from the onboard wireless network is generated by vectorially combining the resulting propagation maps. Results for the two scenarios are presented.
\end{abstract}

\section{INTRODUCTION}

The deployment of wireless communication systems has maintained persistent growth over the last few years [1]. While the portfolio of terrestrial services offered, such as mobile television and wireless internet, has been greatly consolidated, the same cannot be stated for its aeronautical counterpart [2]. As the need for airborne users to be constantly connected to real-time data becomes evermore imperative, researchers in academia as well as in the airline industry, are striving to offer passengers seamless wireless connectivity during flight [3], [4].

Due to the high speeds by which clients travel whilst onboard an aircraft, individual subscriber connections are not feasibly supported [5]. Thus, to facilitate multimedia integrated services, a local area network architecture must be set up onboard large passenger carriers so as to serve as a gateway node to the outside infrastructure. Communications between laptop computers, personal digital assistants, personal electronic devices (PEDs) and the in-flight entertainment (IFE) systems offered onboard aircraft can be offered over the unlicensed industrial, scientific and medical (ISM) at frequency band of $2.4 \mathrm{GHz}$, particularly via the IEEE $802.11 \mathrm{~b}$ standard for wireless local area networks (WLAN). The latter system was principally considered due to its prevalent implementation on modern PEDs and viability for IFE allotment to potential clients onboard the confined space of an aircraft cabin [6].

Several considerations must however precede the implementation of such wireless communication systems. The sum of the electromagnetic fields generated by all the onboard devices present critical issues of electromagnetic interference (EMI), that compromise the safety and integrity of the aircraft's communications and control equipment. These effects emanating from both external and internal electromagnetic (EM) sources [7], have been the reported source for a number of incidents [8]. Public concern was subsequently induced, urging for aircrafts to undergo several certification assessments prior to utilization [9]. Modeling of the radio emissions that penetrate into critical cable enclosures is thus a necessity for the research and development of aircraft manufactures in order to avoid expensive and repetitive measurement campaigns at the design stage [10].

Modeling the propagation of wireless communications in indoor environments is a complex task, since the electromagnetic field at a point is composed from a large number of indirect components [11]. Several approaches have been described in literature for the characterization of electromagnetic phenomena [12]. However, due to their inherent complexity of computation, theoretical methodologies that solve directly Maxwell's Equations, such as Finite-Difference Time Domain [13], [14], or the Methodof-Moments [15], [16], fail to scale up well for implementation in large scenarios. The rapid developments in computer simulation capabilities however, impart the feasibility of implementation of asymptotic techniques such as the case with Ray-Tracing algorithms [17], [18].

Nevertheless, estimating the EMI over the cabin surface is still computationally intensive and a non-trivial task, mainly because different signals arrive via a number of distinct paths. Each of the latter undergoes attenuation from various propagation phenomena such as reflections, refractions, diffractions and scattering within the transmission media [19]. EMI modeling onboard aircraft presents a number of unique characteristics that differ from conventional indoor scenarios. The fuselage construction exhibits a tunnel-like structure, which presents waveguide effects for electromagnetism [20]. On the other hand, the dense cluttering of cabin furniture inside the aircraft significantly inhibits signal propagation whilst generates numerous multipath components [21].

This paper models the EMI present inside the cabin of a large commercial airline, by the application of a three 
dimensional ray-tracing algorithm. With respect to previous cabin implementations [10], [22], which model furniture as planar surfaces, our model enhances the simulation accuracy and detail due to the consideration of volumetric obstacles and subsequent employment of thin film optics physics. A much more realistic result, in comparison to previous simulations, is also achieved from the novel scenario that emulates the interaction from all the potential electromagnetic emitting sources at the demanded frequency range.

This paper is organized as follows; Section II summarizes the ray tracing technique employed whilst providing concise explanations on the various propagation parameters. Section III presents details on the implementation of the algorithm and portrays the attained results. A conclusion in light of the results obtained is finally presented in Section IV.

\section{Propagation Model}

In order to calculate the EMI field incident on the inner fuselage structure of the aircraft, an area oriented design based of the ray launching technique was adopted. This approach encompassed a computational complexity which is quasi independent on the amount of observation points and linearly dependent on the number of subsequent environment interactions [23]. The wavelengths of the signals in the 2.4 GHz ISM frequency band are much smaller than the dimensions of the simulated model. This allows the adoption of the Geometric Optics (GO) hypothesis, whereby EM propagation can be modeled as distinct rays and the latter can be executed separately after each propagation mechanism is individually considered [24].

\section{A. Ray Launching}

The model takes into consideration fixed transmission stations that operate at a defined equivalent isotropic radiated power. Rays from these sources are launched in all relevant directions randomly via a quantized Monte-Carlo method in spherical coordinates employing small angular increments. The latter operates concurrently on the two parameters for spherical directions $\varphi$ and $\theta$, which are bounded by $0 \leq \varphi \leq 2 \pi$ and $0 \leq \theta \leq 2 \pi$ respectively [22]. This ensures that all potential directions for EM field interference are accounted for in an asymptotical manner. In order to avoid the possibility of multiple rays contributing to the same direction, the algorithm was modified as to ensure only unique propagation directions are considered.

The wavefront of the propagating ray is modeled perpendicular to its direction of motion [25]. To account for polarization effects, each ray is separated into parallel and perpendicular components for calculation, denoted by $\|$ and $\perp$ subscripts respectively [26].

As per definition, the basis of ray tracing involves the calculation of the electromagnetic field at any point to be performed by pondering every ray incident on the location [27]. Rays at a single point arrive from a number of directions whilst having experienced several propagation mechanisms, thus the incident power can be expressed as [28]:
$P_{k}=P_{T}\left(\frac{\lambda}{4 \pi r}\right)^{2} \prod_{i} \rho_{i} \prod_{j} \tau_{j}$

where $P_{T}$ represents the transmit power of the antenna in Watts, $\lambda$ is the wavelength in meters, $r$ denotes the unfolded path length in meters, $\rho_{\mathrm{i}}$ and $\tau_{\mathrm{j}}$ are the reflection and transmission coefficients, whilst $i$ and $j$ are the indices that increment over each propagation mechanism respectively. The phase of each component in the multipath summation can be considered as uniformly distributed in the bound $[0,2 \pi)$ since the large amount of scattering components resulting from the dense clutter of obstacles present in the simulated aircraft cabin are uncorrelated [29].

Rays launched from the antenna location inside the cabin environment, are eventually incident on either the fuselage structure or the aircraft furniture. Subsequently each ray undergoes a number of propagation mechanisms to eventually generate multipath EMI. This depends on the objects encountered, the electromagnetic parameters of the materials, and the angle and power of the incident rays.

\section{B. Reflection and Transmission}

Adhering to the requirements set by the GO hypothesis; surface boundaries are defined as two separate infinite half spaces, each having different permeability, permittivity and conductivity attributes and are divided by a smooth planar interface [30]. The product coefficients of reflection and transmission that result when an incident ray collides with such a boundary are derived by employing a simplification of Maxwell's Equations at boundary conditions, commonly referred to as Fresnel's Equations and defined by:

$$
\begin{aligned}
& T_{\perp}=\frac{2 \mu_{t} \eta_{i} \cos \theta_{i}}{\mu_{t} \eta_{i} \cos \theta_{i}+\mu_{i} \eta_{t} \cos \theta_{t}} A_{\perp}, \\
& R_{\perp}=\frac{\mu_{t} \eta_{i} \cos \theta_{i}-\mu_{i} \eta_{t} \cos \theta_{t}}{\mu_{t} \eta_{i} \cos \theta_{i}+\mu_{i} \eta_{t} \cos \theta_{t}} A_{\perp}, \\
& T_{\|}=\frac{2 \mu_{t} \eta_{i} \cos \theta_{i}}{\mu_{i} \eta_{t} \cos \theta_{i}+\mu_{t} \eta_{i} \cos \theta_{t}} A_{\|}, \\
& R_{\|}=\frac{-\mu_{i} \eta_{t} \cos \theta_{i}+\mu_{t} \eta_{i} \cos \theta_{t}}{\mu_{i} \eta_{t} \cos \theta_{i}+\mu_{t} \eta_{i} \cos \theta_{t}} A_{\|} .
\end{aligned}
$$

where $\mathrm{A}$ is the power of the incident ray on the boundary, subscripts $i$ and $t$ represent the medium in which incidence and transmission will occur respectively. The permeability of each material is expressed by $\mu, \theta$ symbolizes the angle of the ray with the vector normal to the point of intersect, whilst $\eta$ epitomizes the complex refractive index of the boundary media defined by:

$$
\eta=\sqrt{\mu \varepsilon}+i \frac{c}{\omega \delta}
$$

where $\varepsilon$ represents the permittivity of the material, $\omega$ is the angular frequency, and $\delta$ is the skin depth of the boundary 
material articulated as the reciprocal of the attenuation constant. The latter parameter, symbolized by $\alpha$, is calculated using [25]:

$$
\alpha=\frac{1}{\delta}=\omega\left[\frac{\mu \varepsilon}{2}\left[\sqrt{1+\left(\frac{\sigma}{\omega \varepsilon}\right)^{2}}-1\right]\right]^{\frac{1}{2}},
$$

with $\sigma$ denoting the conductivity of the material. In order to enhance the accuracy of the model, the structure components of the cabin are simulated with their actual thickness. This consideration demanded the adaptation of the physical principles employed in thin film optical media together with GO in order to account for the effects generated by parallel boundaries of alternating media. The Fresnel's equations for transmission and reflection respectively, had also to be modified as to account for non-normal incidence on boundaries with reciprocal complex refractive index ratios. The compensation equations, that integrate the cross-sectional area upon which EM intensity is measured, are given by [31]:

$R=\frac{|R|^{2}}{|A|^{2}}$, and $T=\frac{\eta_{t} \cos \theta_{t}|T|^{2}}{\eta_{i} \cos \theta_{i}|A|^{2}}$

Additionally, a phase shift of $\pi$ is also inferred to the reflected ray in order to accurately account for interference [32]. The directions by which the reflected and transmitted rays propagate are derived by employing the mathematics of three-dimensional vectors in [33]:

$$
\begin{aligned}
& R=2(N \cdot I) N-I, \\
& T=\left(\frac{\eta_{i}}{\eta_{t}} N \cdot I-\sqrt{1-\frac{\eta_{i}^{2}}{\eta_{t}^{2}}\left[1-(N \cdot I)^{2}\right]}\right) N-\frac{\eta_{i}}{\eta_{t}} I,
\end{aligned}
$$

where $I$ represents vector incident on the boundary, $N$ is the $3 \mathrm{D}$ normal vector to the plane and $(\cdot)$ refers to the dot product operation between vectors.

\section{Scattering}

Propagation of waves due to scattering was further added to the model. This addition enhances the accuracy and validation of the attained results. The latter removed the assumption of considering reflection off surfaces from being solely specular [34]. In a pragmatic scenario, a factor of the energy reflected in the same medium of incidence is scattered in alternative directions other than the specular via coherent and incoherent components. This occurs if the mean value of the protuberances in the height values of the incident boundary exceeds the heuristic Rayleigh criterion expressed by [35]:

$\sigma_{h}<\frac{\lambda}{8 \cos \theta_{i}}$

Other than the aluminium fuselage, all the surfaces with which rays are incident inside the cabin are defined as slightly rough in nature and contribute the scattering phenomenon. Assuming that the variance of surface heights obeys a Gaussian distribution having no sharp edges and shadowing effects, the reduction on the power density of the reflected ray due to the scatter loss factor can be derived as [36]:

$\rho_{s}=e^{-8 \pi\left(\frac{\sigma_{h}}{\lambda}\right)^{2} \cos ^{2} \theta_{i}}$.

The effects of diffraction were not considered in the design of the algorithm due to its use in the $2.4 \mathrm{GHz}$ band that renders its contribution negligible compared to the other propagation characteristics. Also, in locations such as the aircraft cabin, where the separation between transmitter and receiver is not too large, the effects of diffraction further loose their significance [38], [39].

\section{IMPLEMENTATION AND SIMULATION RESULTS}

The algorithm developed in this paper was used to model the EMI onboard a large furnished commercial aircraft generated by the use of PED communication together with network hubs. The aircraft model replicates a commercial 362 passenger Airbus A380-600 aircraft. Simulated were performed using Matlab ${ }^{\circledR}$ with spatial resolution of $10 \mathrm{~cm}$. A three-class cabin layout was adopted with 54 business class locations and 308 economy seats. The airliner was composed mainly from an aluminum fuselage with galleys to isolate the three-class sections. The inside cabin, was furnished with wooden flooring, rows of leather seats, and stowage compartments on the ceiling. The material parameters used in the model are defined in Table 1.

TABLE I

Electromagnetic Material Properties

\begin{tabular}{|l|c|c|c|c|}
\hline \multirow{2}{*}{ Parameters } & \multicolumn{4}{|c|}{ Material } \\
\cline { 2 - 5 } & Air & Seat & Wood & Aluminum \\
\hline Permittivity $(\varepsilon)$ & 3 & 3 & 3 & 1.0005 \\
\hline Permeability $(\mu)$ & 1 & 1 & 1 & 1 \\
\hline $\begin{array}{l}\text { Electrical } \\
\text { Conductivity }(\sigma)\end{array}$ & $5 \mathrm{e}^{-15}$ & 0.01 & 0.01 & $4 \mathrm{e}^{7}$ \\
\hline
\end{tabular}

To generate the propagation map inside the cabin, a large number of rays are emanated sequentially from the source locations, as described in Section IIA, until most of the relevant directions are accounted for in an asymptotical manner. Each ray undergoes free space propagation until it impinges on a cabin component. Consequently, the EM field of the ray is vectorially summed with the EMI already present on the respective point from previous incident multipath components. Depending on the surface boundary incident, the ray is either totally reflected in the same medium or decomposed into reflected and transmitted components. Due to the frequency range considered by the model and the high conductivity of aluminum at such frequencies, the condition of total internal reflection occurs only in case of collision of the incident ray with the fuselage.

Following an interaction with a medium boundary, the propagation of the reflected and transmitted rays are 
subsequently simulated until they either collide with another boundary or attenuate to more than $-150 \mathrm{dBm}$, at which point their EMI contribution is negligible. Rays traveling inside a medium experience a loss function proportional to the permittivity, electrical conductivity and distance propagated until they interact with another boundary. Thus, this generates a scenario whereby a residue of a transmitted ray continues to propagate inside a medium bounded by discontinuity boundaries due to reflected component as dictated by the physics of thin film optics and depicted in Fig. 1.

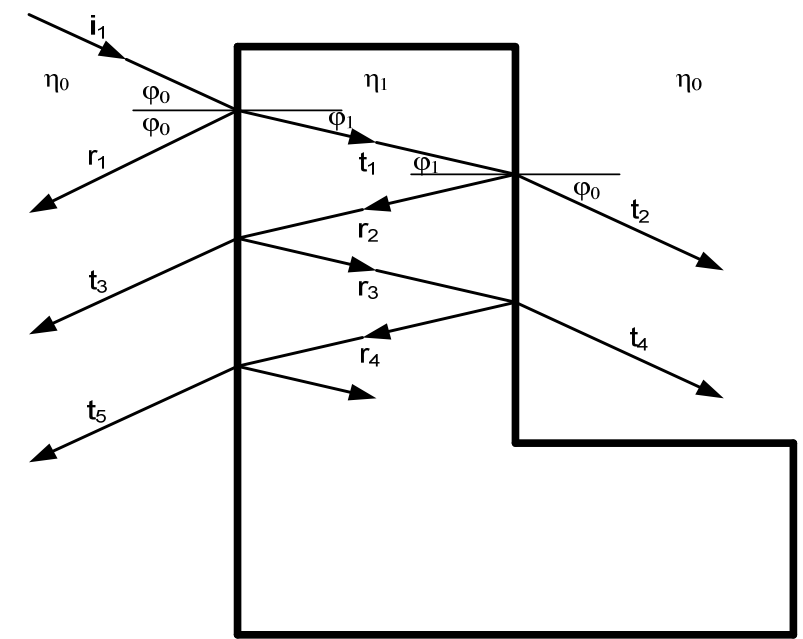

Fig. 1. Propagation of rays inside cabin seating modeled as thick media bounded by the air medium.

The WLAN considered takes into account the different channels employed by the IEEE 802.11 b standard, which ranges from $2.412 \mathrm{GHz}$ to $2.462 \mathrm{GHz}$ at regular intervals of $5 \mathrm{MHz}$ and accurately maintains a separate set of data for each particular frequency. Two separate scenarios were simulated by the algorithm that study the effects generated by servicing the entire aircraft network from; (a) A single WLAN base station or (b) by distributing the load over four strategically placed wireless access points. Setup (a) simulated 200000 rays launched from the antenna that was centrally located just under the stowage bays and characterized by an equivalent isotropically radiated power (EIRP) of $45 \mathrm{dBm}$. This generated an EM coverage map as depicted in Fig. 2a. Simulation (b) involving distributed base stations for network coverage, shown in Fig. 2b, satisfied the minimum detectable signal criterion in all locations of the aircraft with an EIRP power of just $30 \mathrm{dBm}$. As can be deduced from Fig. 2, the amount of EMI incident on the fuselage from the second configuration, although uniformly spread across the whole fuselage, is much less critical for interference, since the levels of EM fields are weaker. This is mainly attributed to the fact that there is no need for the WLAN stations to transmit at high power to satisfy the coverage criterion.

As can be noted from Fig. 3, the power delivered by the transmitting antenna is severely attenuated once the line-ofsight is impeded and the ray is incident on the furniture of the structure. Nevertheless, Fig. 2 indicates that the multipath contribution generated from the dense clustering present in the aircraft environment still presents a significant EMI field in shadowed locations of the aircraft.

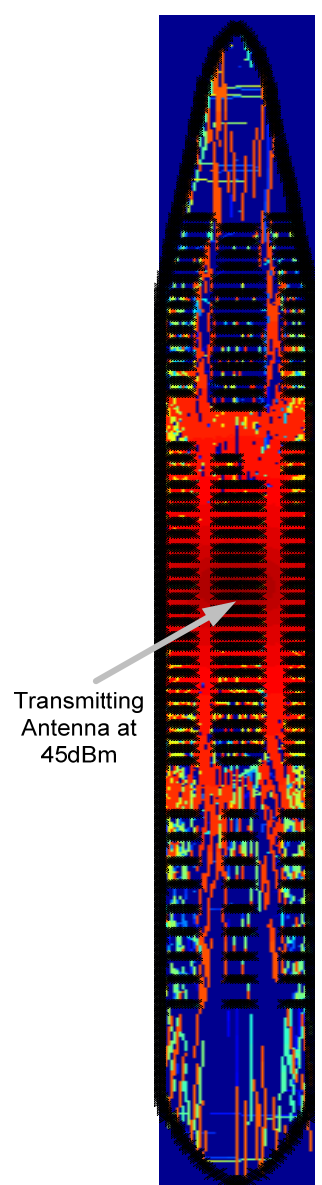

(a)

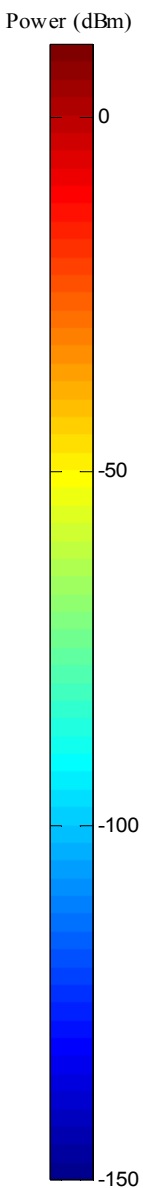

(b)

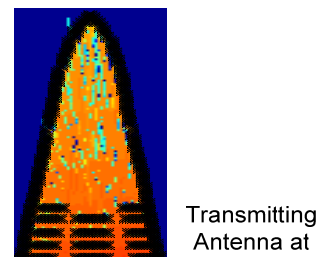

Antenna at

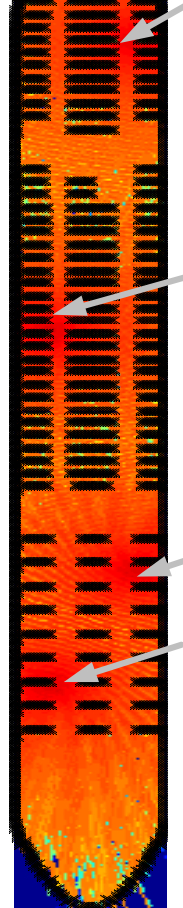
$30 \mathrm{dBm}$

Fig. 2. EM coverage map for the entire fuselage from a) one central transmitting node at $32 \mathrm{~W}$, b) four strategically located antennas transmitting at $1 \mathrm{~W}$ each.

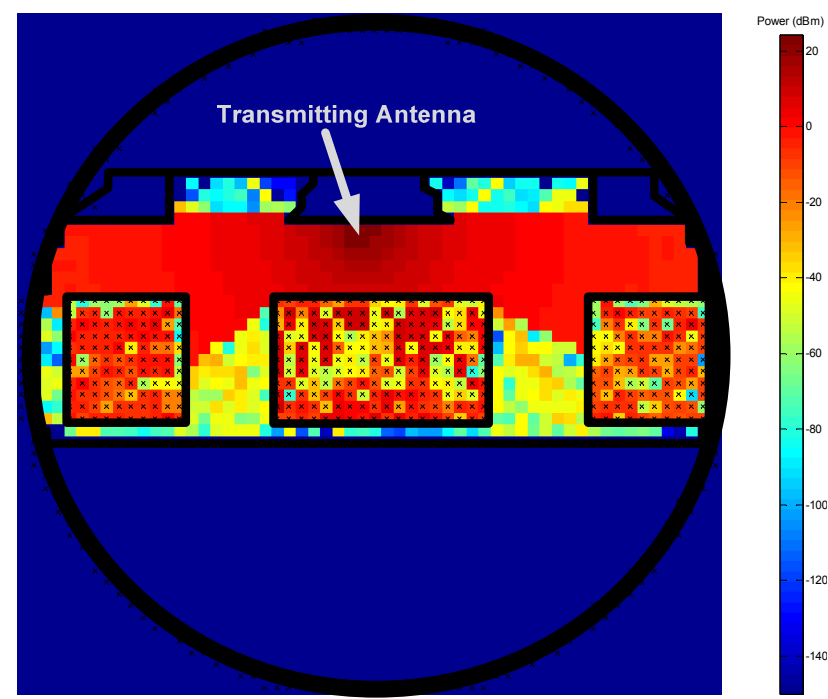

Fig. 3. Fuselage cross section depicting the propagation map radiated from the antenna and EM attenuation suffered due to obstacle interference. 

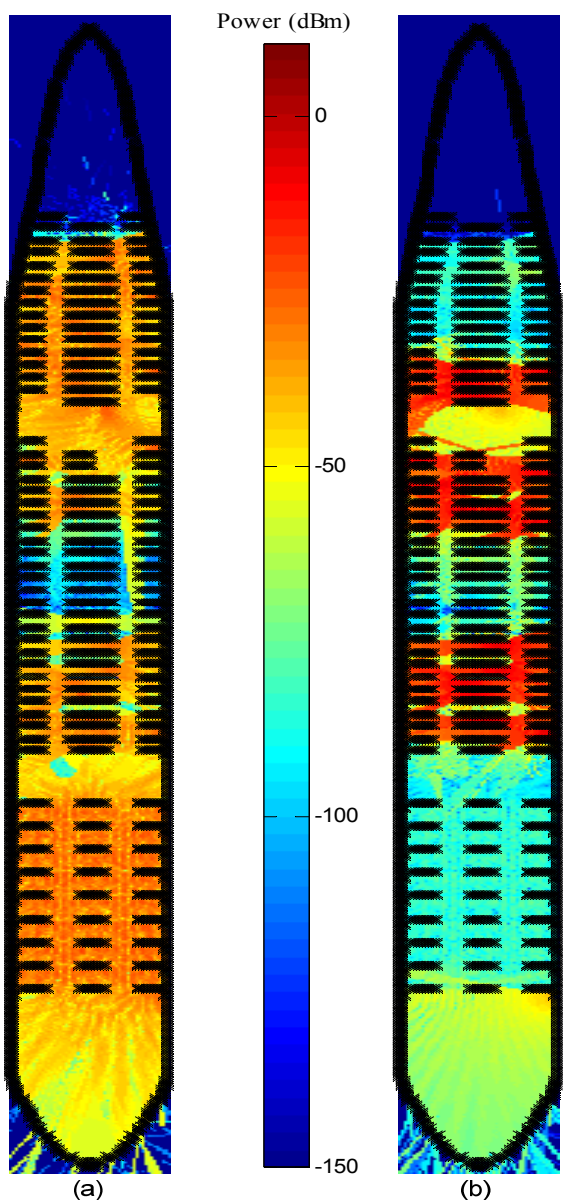

(b)

Fig. 4. Electromagnetic Interference incident on aircraft due to uplink communication from PEDs.

To provide a more holistic understanding of the EMI experienced by the aircraft, 120 clients interacting via their PEDs with the network are simulated. All business class passengers are connected to the WLAN while a spatially randomly distributed $20 \%$ from the economy class commuters makes use of the network. Subsequent to computing the EM propagation map emitted by the WLAN base stations inside the cabin, the EM field present at the receiver locations is employed as data to calculate the power by which user nodes would require to transmit data on the uplink. The mobile PEDs were simulated to transmit in the Class 3 power range with a potential maximum power of $24 \mathrm{dBm}$, whilst having a minimum detectable signal of $-117 \mathrm{dBm}$ to sustain a transmission with mean bit error rate of $10^{-3}$. Due to the IEEE $802.11 \mathrm{~b}$ protocol, the PEDs communication could be performed on any of the eleven allocated channels in the $2.4 \mathrm{GHz}$ range. Fig. 4 displays the average EMI generated on the uplink channel of the network, for randomly distributed devices in both scenarios. The results depicted in Fig. 2 and Fig. 4 and discussed above confirm that the PEDs transmit at a higher power when located at points characterized by a weak downlink signal from the base station. Apart from reducing the lifetime of mobile devices, this result implies that a greater interference field is incident on the fuselage. The severe EM interference patterns that can be comprehended in Fig. 5, portray the total EMI incident from the network onto the aircraft structure. It is thus evident that a system involving distributed base stations, although minimally increasing weight, presents a much lower EMI field and should be considered as the better solution. Nevertheless, the need for the development of better shielding is clear. This is even more critical for cables and systems employed for communications and control.

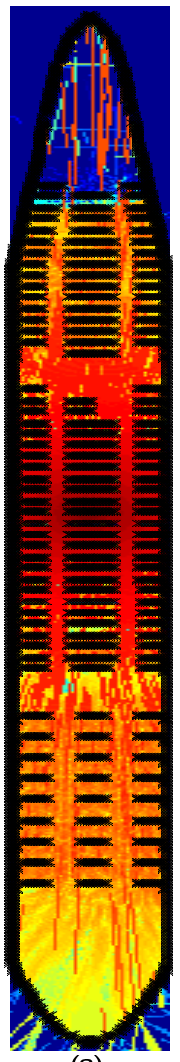

(a)

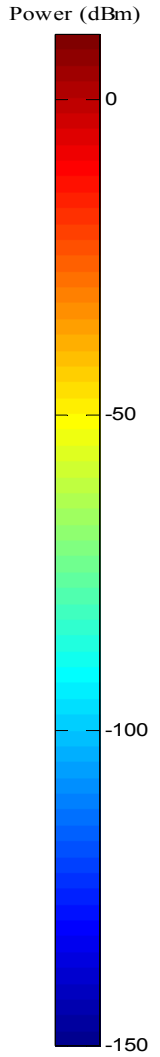

500

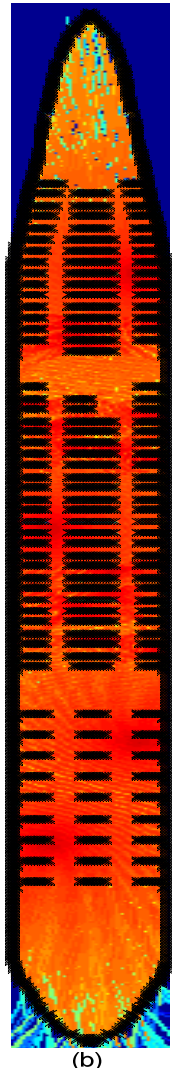

Fig. 5. Electromagnetic Interference incident on aircraft due to WLAN base stations and personal electronic devices.

Furthermore, the model was developed in a modular manner to allow for feasible adaptation and scalability. This is of particular interest to the aircraft industry due to large permutations of unique cabin layouts demanded by airlines. The model should therefore, help in reducing the expensive and time consuming measurement iterations that are otherwise required during design time, to ensure compatibility with the EM standardization.

\section{CONCLUSION}

This paper describes a ray-tracing based model that is used to calculate the EMI generated inside an aircraft cabin. The interference levels caused by the deployment of a WLAN network on a large commercial airline were computed. The model includes the effects of multipath electromagnetic propagation that results from the relatively small cabin crosssection as well as from the dense furniture onboard. The EMI 
field computed is due to the summation of the fields incident from the base stations and a number of randomly located PEDs onboard the aircraft. The attained results provide an allencompassing understanding of the generated EMI and suggest the implementation of distributed base stations around the aircraft at lower transmission power to alleviate the issue, whilst offering support for the development of conscientious cable design in aircraft.

\section{ACKNOWLEDGMENT}

This work forms part of the project HIRF SE which is financially supported under the European Union 7th Framework Programme (FP7). The authors are solely responsible for the contents of the paper which does not represent the opinion of the European Commission.

\section{REFERENCES}

[1] M.C. Lawton and J.P. McGeehan, "The application of GTD and ray launching techniques to channel modeling for cordless radio systems," in Proc. of 42nd IEEE Vehicular Technology Conf., pp. 125-130, Denver, 1992.

[2] N.R. Diaz and J.E. Esquitino, "Wideband channel characterization for wireless communications inside a short haul aircraft," in Proc. of the 59th IEEE Vehicular Technology Conf., pp. 223-228, Germany, May 2004.

[3] A. Jahn, et. al. "Evolution of aeronautical communications for personal and multimedia services," in IEEE Communications Magazine, Vol. 41, pp. 36-43, July 2003.

[4] M. Werner and M. Holzbock, "System Design for Aeronautical Broadband Satellite Communications", in Proc. of IEEE International Conference on Communications, pp.2994-2998, May 2002.

[5] K.D. Lin and J.F. Chang, "Communications and entertainment onboard a high-speed public transport system", in IEEE Wireless Communications, Vol. 9, pp84-89, Feb. 2002.

[6] D.B. Lee, "In-flight entertainment-getting from wishlist to reality", in Proc. of the 17th Digital Avionics Systems Conf., pp. G16/1-G16/8, USA, Nov. 1998.

[7] K.W. Hurst and S.W. Ellingson, "Path loss from a transmitter inside an aircraft cabin to an exterior fuselage-mounted algorithm," in IEEE Trans. on Electromagnetic Compatibility, Vol. 50, No. 3, pp. 504-512, Aug. 2008.

[8] S.V. Georgakopoulos, C.R Birtcher, C.A. Balanis and R.A. Renaut, "HIRF penetration and PED coupling analysis for scaled fuselage models using a hybrid subgrid $\operatorname{FDTD}(2,2) / \operatorname{FDTD}(2,4)$ method," in IEEE Trans. on Electromagnetic Compatibility, Vol. 45, No. 2, pp. 293-305, May 2003.

[9] C.R. Paul, Introduction to Electromagnetic Compatibility, 2nd ed., John Wiley and Sons, New York, 1992.

[10] K. Chetcuti, C.J. Debono, R.A. Farrugia and S. Bruillot, "Wireless propagation modelling inside a buisness jet", in Proc. of Eurocon 2009, pp. 1640-1645, May 2009..

[11] A. Neskovic, N. Neskovic and G. Paunovic, "Modern approaches in modeling of mobile radio systems propagation environment," in IEEE Communications Surveys, pp. 2-12, third quarter, 2000.

[12] M.C. Lawton and J.P. McGeehan, "The application of a deterministic ray launching algorithm for the prediction of radio channel characteristics in small-cell environments," in IEEE Trans. on Vehicular Technology, Vol. 43, No.4, pp. 955-969, 1994.

[13] K.S. Yee, "Numerical solution of initial boundary value problems involving Maxwell's equations in isotropic media," in IEEE Trans. on Antennas and Propagation, vol. 14, no. 3, pp. 302-307, 1966.

[14] A. Taflove, Computational Electrodynamics, The Finite-Difference Time-Domain Method, Artech House, Boston, 1995.

[15] R.F. Harrington, Field Computation by Moment Methods, Macmillan, New York, 1968.

[16] R. Mittra, Numerical and Asymptotic Techniques in Electromagnetics" Springer Verlag, New York, 1975.
[17] K.H. Ng, E.K. Tameh, A. Doufexi, M. Hunukumbure and A.R. Nix, "Efficient multielement ray tracing with site-specific comparisons using measured MIMO channel data," in IEEE Trans. on Vehicular Technology, Vol. 56, No. 3, pp. 1019-1032, May 2007.

[18] J.S. Seybold, Introduction to RF propagation, Wiley, New Jersey, 2005.

[19] L. Deryck, "Natural propagation of electromagnetic waves in tunnels," in IEEE Trans. on Vehicular Technology, Vol. 27, No. 3, pp. 145-150, 1978.

[20] N.R. Diaz and M. Holzbock, "Aircraft cabin propagation for multimedia communications," in 5th European Mobile and Personal Satellite Communications Workshop, Italy, September 2002.

[21] A. Kaouris, M. Zaras, M. Revithi, N. Moraitis and P. Constantinou, "Propagation measurements inside a B737 aircraft for in-cabin wireless networks," in Proc. of IEEE Vehicular Technology Conf., pp. 29322936, Singapore, May 2008.

[22] N.R. Diaz and C. Achilli, "Cabin channel characterization for personal communications via satellite," in Proc. of 21st International Communications Satellite Systems Conf. and Exhibit, April 2003.

[23] B.E. Geschwendtner, G. Wolfle, B. Burk and F.M. Landstrofer, "Ray tracing vs. ray launching in 3-D microcell modeling" in Proc. of the European Personal and Mobile Communications Conf., pp 74-79, 1995.

[24] J. Lacik, Z. Lukes and Z. Raida, "On using ray-launching method for modeling rotational spectrometer," in Radioengineering, Vol.17, No.2, pp.98-107, June 2008.

[25] F.T. Ulaby, Electromagnetics for Engineers, Pearson International Edition, Prentice Hall, 2005.

[26] C.J. Debono and R.A. Farrugia, "Optimization of the UMTS network radio coverage on-board an aircraft," in Proc. of the IEEE Aerospace Conf., March 2008.

[27] K.W. Cheung and R.D. Murch, "Measurement, characterization and modeling of the wideband indoor channel," in Proc. of the 46th IEEE Vehicular Technology Conf., pp 588-592, Atlanta, April 1996.

[28] S. Fortune, "Algorithms for prediction of indoor radio propagation," technical report, Bell Laboratories, 1998.

[29] M.C. Lawton, R.L. Davies and J.P. McGeehan "A ray launching method for the prediction of indoor radio channel characteristics" in IEEE Symposium on Personal, Indoor and Mobile Radio Communications, pp 104-108, Sep 1991.

[30] D.Didascalou, "Ray-optical wave propagation modelling in arbitrarily shaped tunnels", Ph.D. dissertation, Univ. of Karlsruhe, Germany, 2000.

[31] F.A. Jerkins and H.E. White, Fundamentals of Optics, 4th Ed., McGraw-Hill, 2003.

[32] M. Born and E. Wolf, Principles of Optics, 7th Ed., Cambridge University Press, Cambridge, 1999.

[33] E. Lengyel, Mathematics for 3D Game Programming and Computer Graphics, 2nd Ed., Charles River Media, 2003.

[34] F. Layer, R. Kattenbach and H. Fruchting, "Ray optical approach for wideband indoor wave propagation model with moving reciever," in IEEE 8th International Symposium on Personal, Indoor and Mobile Radio Communications, pp. 135-139, Helsinki, September 1997.

[35] L. Boithias, Radio Wave Propagation, McGraw-Hill Inc., New York, 1987.

[36] O. Landron, M.J. Feuerstein and T.S. Rappaport, "In Situ Microwave Reflection Coefficient Measurements for Smooth and Rough Exterior Wall Surfaces", IEEE Trans. in Vehicular Technology, Vol. 51, No. 4, pp. 1251-1258, July 2005.

[37] D.J. Cichon, "Ray optical modeling of wave propagation in urban micro- and picocells", Ph.D. dissertation, Univ. of Karlsruhe, Germany, 1994.

[38] J.M. Espinosa, J.M. Hernando, F.M. Perez, J.A Milanes, L. Hidago and M. Gallardo, "Coverage simulator for wireless networks" in IEEE International workshop on Factory Communication Systems, pp 149156, Barcelona, Spain 1997.

[39] D. Chizhik, J. Ling, R.A. Valenzuela, "The effect of electric field polarization on indoor propagation," in IEEE International Conf. on Universal Personal Communications, pp 459-462, Italy, October 1998. 